1. Ventricular tube must be inserted from the burr hole opened at the occipital region in the postero-anterior direction long enough through the lateral ventricle.

2. The flushing device must be applied to every case. We can not only put away various obstructors, but also we can know the point of obstruction. If return of the dome of the flushing device is incomplete, the point of obstruction is in the ventricular end. And if we feel resistance by compression of the dome, obstruction is in the cardiac end.

3. The cardiac tube must be fixed as higher as possible in the right atrium, in order to prevent the destruction of the tricuspid valve and the cardiac tube. In order to determine the length of the cardiac tube, we adopt the three indicators as follows;

1. Chest X-Ray.

2. EKG.

3. Micro drip chamber.

To prevent skin necrosis over the tube or the flushing device, it is very important to close the operation wound with two layers. Protein anabolic hormone is effective in case of malnutrition.

\title{
21. Ventriculosagittal Sinus Shunt in Hydrocephalus
}

\author{
Shunshiro Kondo, Susumu Sato, Kazuo Aoyama and Minoru Endo \\ Department of Neurosurgery, The Tokyo Rosai Hospital
}

A new type of the shunt operation was devised first by Paul C. Sharkery in 1964 for the chronic hydrocephalus by of Ventriculosagittal sinus shunt. The experience of this type of operation in 5 patients will be presented.

The shunting system consisted of ventricular tube, flushing device and Pudenz-Heyer tube and the right ventricle was tapped through a burr hole and ventricle tube was inserted into the ventricle and this was connected to the flushing device seated on the burr hole. Next, a small incision was made on the sagittal sinus through another burr hole and Pudenz-Heyer tube was inserted in the sinus towards $\mathrm{lcm}$. distal to the confluens sinuum and this was connected to the flushing device.

The lengh of the venous tube was determined beforehand by measuring the distance between the expected first burr hole and the confluens sinuum in the X-ray film. Moreover, the tip of venous tube was adjusted during operation by using image intensifier. 
The advantage of this technique compared with the ordinary ventriculo auriclar shunt seemed to be 1) lesser displacement of tip of venous tube by necl movement or by growth of the body 2) no need of special check on heart durin: operation.

All 5 subjected patient under this technique had communicating hydro cephalus with high intracranial pressure. Postoperatively, these patients shower continuous effect of the shunting system and showed clinical improvement, i.e. lessened convulsion, spasticity in extrimity, intracranial pressure, headache or bette mental state, activeness and improved E.E.G. findings .

Complications such as mechanical failure, thrombosis or septicemia were no encountered in the series.

\title{
22. Experimental Studies on Blood in Subarachnoid Space
}

\author{
Kazuo Usui, Yoshio Hashimoto and Kinjiro Iwata \\ First Surgical Department, Nagoya University School of Medicine
}

A genesis of hydrocephalus following subarachnoid hemorrhages was ex perimentally studied. Autogenous whole blood, suspension of red blood cells plasma and hemolysed red blood cells were injected repeatedly in the cistern: magna of adult rabbits. Volumes or amounts injected were 1.3-2.0 cc. of whol blood, suspension of red blood cells washed from 1.5-2.0 cc. of whole blood 0.8-1.7 cc. of plasma, and hemolysed red blood cells aquired from 1.0-1.5 cc. o whole blood (washed in saline and hemolysed in distilled water). Intervals o injections were mostly 7-8 days but up to 21 days in some rabbits. Sacrific and autopsy were done 7-20 days after the last injections. Dilatation of th cerebral ventricles was recognized with high incidence (7/9) only in the group o rabbit which received more than 3 times of injections of whole blood and not i ones of other groups. Hydrocephalus was of communicating type accompaine with widening of the aqueduct. Ferric iron reaction revealed hemosiderin-deposit in fibrosed or organized leptomeninges and in subpial layers of the basal cortex 0 the rabbits injeted with whole blood or suspension of red blood cells. It is postu lated that there must be clot formation and its organization in subarachnoid spac which in turn requires fibroblastic reactions of the leptomeninges induced by irritan actions of some blood components. Intracisternally injected hemolysed red bloo cells had peculiar effect; generalized convulsive seizures with opisthotonus an tonic extensions of all extremities were observed at or some seconds after th injection. These convulsive seizures often resulted in acute death or extrem emaciation of the animals. In this respect, it seems very important whethe 